\title{
NEURALGIC AMYOTROPHY
}

\author{
KENNETH J. FAVERO, ROBERT H. HAWKINS, MICHAEL W. JONES
}

\author{
From Shaughnessy Hospital, Vancouver
}

\begin{abstract}
Thirty-nine patients with neuralgic amyotrophy were reviewed. In addition to the clinical findings reported in the neurological literature, we commonly found pain throughout the upper limb, and diffuse involvement of the brachial plexus. Five patients had spinal accessory nerve lesions and five had glenohumeral instability. Sixty-four per cent of the patients had had an orthopaedic consultation during their acute illness. It is therefore important that orthopaedic surgeons are aware of this clinical syndrome and its management. The prognosis is excellent with non-invasive treatment.
\end{abstract}

Neuralgic amyotrophy (brachial plexus neuropathy, brachial plexus neuritis, or Parsonage-Turner syndrome) was first recognised in the 1940 s, but there has been little progress since then in understanding this clinical enigma. Although it is well described in both medical and neurological publications, there are few references in the orthopaedic literature.

The term neuralgic amyotrophy is used throughout this paper, and is suggested as the best name for the disorder, since it has not been proven that a "neuritis" is the cause, and "neuropathy" is too general a term. In addition, involvement of a nerve outside the brachial plexus, such as the spinal accessory nerve, may occur.

The syndrome was first recognised as a distinct clinical entity by Parsonage and Turner in 1948, who described its presentation with sudden onset of severe pain affecting the shoulder girdle, followed by the rapid development of weakness and atrophy. Fasciculation was rare, but cutaneous sensory loss, especially in the distribution of the circumflex nerve, was not uncommon. Although it was usually unheralded, the condition was reported to have occurred after viral infections, vaccinations, and surgery. Bilateral disease was recognised, with either symmetric or asymmetric involvement. Recurrence was rare (Turner and Parsonage 1957) and the aetiology unknown. Some authors reported an epidemic form of the disease (Wyburn-Mason 1941), while others discussed a rare familial presentation (Widerholt 1974;

K. J. Favero, MD, Orthopaedic Resident University of British Columbia

R. H. Hawkins, MD, FRCS(C), Orthopaedic Surgeon

$M$. W. Jones, MD, FRCP(C), Neurologist

Shaughnessy Hospital, 4500 Oak Street, Vancouver, British Columbia, Canada V6H 3N1.

Requests for reprints should be sent to Dr R. H. Hawkins.

(C) 1987 British Editorial Society of Bone and Joint Surgery 0301-620X/87/2062 \$2.00
Bradley et al. 1975; Dunn, Daube and Gomez 1978). It may be caused by an inflammatory process of either infectious or allergic origin (James and Miles 1966), but the possibility that there are several different diseases resulting in one syndrome has been noted (Bradley et al. 1975).

Electrophysiological or electromyographic (EMG) studies are frequently used to confirm the diagnosis. Findings include abnormal sensory potentials, lack of paraspinal denervation potentials, and abnormal distal conduction velocities (Flaggman and Kelly 1980). These changes may indicate demyelinisation ( $\mathrm{O}^{\prime}$ Brien and Payan 1980), or severe axonal damage (Lancet 1980). If studies are performed bilaterally, involvement is frequently shown to be bilateral although this may not have been clinically apparent (Tsairis, Dyck and Mulder 1972).

The prognosis is good (Turner and Parsonage 1957; James and Miles 1966; Lancet 1980). Tsairis et al. (1972) who studied 99 patients with neuralgic amyotrophy concluded that this disease carried an excellent prognosis, with functional recovery in $80 \%$ of patients at two years after onset, and in $90 \%$ at three years. It appears that more distal lesions tend to recover more quickly than more proximal lesions (James and Miles 1966), and upper plexus lesions seem to recover more quickly than lower plexus lesions (Tsairis et al. 1972).

Few articles on this subject have been found in the English language orthopaedic literature: in 1976, Bacevich described one patient with neuralgic amyotrophy and radiological evidence of glenohumeral subluxation; and in 1983, Foo and Swann reviewed a series of 20 patients with isolated paralysis of the serratus anterior and ascribed most of these cases to neuralgic amyotrophy. There have been articles that describe operative procedures, but these would be more appropriate for poliomyelitis than for a syndrome with an excellent prognosis following non-invasive management. 
The purpose of this paper is to describe the clinical findings in a group of 39 patients, in order to familiarise the orthopaedic surgeon with the clinical syndrome and its management.

\section{METHODS AND MATERIALS}

The medical records and electrophysiological studies of all patients with neuralgic amyotrophy seen between July 1976 and April 1985, at Shaughnessy Hospital in Vancouver, British Columbia, were retrospectively reviewed. All 39 patients were personally examined by the authors and all were found to have had historical, clinical, or electrophysiological evidence of the syndrome at the time of their initial consultation. A further 11 patients, initially considered for the study, were excluded since they ultimately had diagnoses other than neuralgic amyotrophy.

\section{RESULTS}

There were 29 men and 10 women in the study group. The mean age was 43 years, with a range of 20 to 71 years.

Thirty-seven patients gave a typical history of sudden onset of severe pain affecting an upper limb, followed by weakness and atrophy as the pain subsided. Seventy-nine per cent had pain at night, or awoke in the morning with pain. Most patients had various combinations of neck, shoulder, arm, and hand pain. In most, the pain persisted for one to four weeks.

There were 34 unilateral and five bilateral lesions. Of the unilateral lesions, the right side was involved approximately three times more frequently, and this was unrelated to sex, occupation, or handedness.

Lesions involved the upper brachial plexus in 22 cases $(50 \%)$, while diffuse plexus involvement was seen in 14 cases $(32 \%)$. Lower and posterior plexus lesions were less common. One case involved the spinal accessory nerve in isolation. There were six recurrent plexus lesions in 44 shoulders, not always involving the same nerve distribution. The time between episodes ranged from 3 to 18 months, and averaged 8 months.

Neurological findings were analysed in four categories (Table I).

Table I. Summary of neurological findings

\begin{tabular}{llll}
\hline Type & Changes noted & Frequency & Percentage \\
\hline Motor & Weakness and paralysis & 37 of 39 & 95 \\
Sensory & Objective abnormalities & 13 of 49 & 27 \\
Reflexes & Depressed or absent & 15 of 49 & 31 \\
EMG studies & Abnormalities & 29 of 35 & 83 \\
\hline
\end{tabular}

Motor changes. Weakness and paralysis were present in 37 patients at initial examination; the other two had recovered but had a typical history and corroborative electrophysiological studies. Single peripheral nerve involvement was seen in eight patients and multiple peripheral nerves were involved in the remainder. The long thoracic and suprascapular nerves were commonly affected together. The most commonly involved muscle groups were the serratus anterior and spinati, followed by the deltoid, triceps, and the wrist and finger extensors. Fasciculation was rare and seen in only two patients.

Sensory abnormalities. Objective sensory abnormalities were present in 13 of the 49 plexus lesions.

Reflexes. Depressed or absent reflexes were present in 15 of the involved upper extremities.

Electrophysiological studies. EMG abnormalities were present in 29 of the 35 patients tested. These consisted of fibrillation potentials, sharp peak positive waves, or prolonged polyphasic action potentials.

In addition, glenohumeral subluxation was found on clinical examination in four patients but this subsequently resolved. Winging of the scapula was present in 12 cases. Radiological examination of the neck was conducted in 27 patients and minor degenerative changes were found in seven. Ten patients had a history of viral infections or surgery, but none followed vaccination.

Twenty-five of the 39 patients $(64 \%)$, had an orthopaedic consultation at some stage during their acute illness.

\section{CASE REPORTS}

Case 1. A 60-year-old right-handed man developed pneumonia and received oral antibiotic treatment as an outpatient. Two weeks after recovery, he experienced sudden, spontaneous onset of severe pain in the right shoulder. For the first two nights the pain was so intense he could not sleep, and during this time he noticed gradual onset of weakness in his arm on attempting to reach upwards or sideways. Three or four days later, he developed lateral numbness in the arm. He resorted to shaving with two hands and holding a coffee cup with his right forearm supported. When seen a month later (Fig. 1), the intense pain had resolved, but the weakness and numbness persisted. Involvement of the axillary (motor and sensory) and suprascapular nerves was confirmed on examination. Substantial recovery had taken place by two months after onset.

Case 2. A 27-year-old right-handed man had a four-year history of recurrent anterior subluxation in his left shoulder, often with trivial insult such as opening a curtain or reaching into a closet for shoes. He awoke one morning with his shoulder displaced and reduced it himself. He was mildly uncomfortable afterwards, but noticed no numbness or weakness. Two days later, his shoulder suddenly became excruciatingly painful and he could not sleep that night. Shortly afterward he noticed increasing weakness of wrist extension and later numbness in the dorsal web space between the thumb and index finger. Examination revealed profound weakness of wrist and finger extensors (Fig. 2), and moderate weakness in deltoid, biceps, triceps, and the intrinsic muscles of the fingers. Anaesthesia was present in the first dorsal web space (Fig. 3). Treatment was supportive, but six months later, when there was still no recovery of wrist extension, he continued to wear a wrist-drop splint. There was some recovery from numbness in the hand, and power had returned in the other involved muscle groups. 


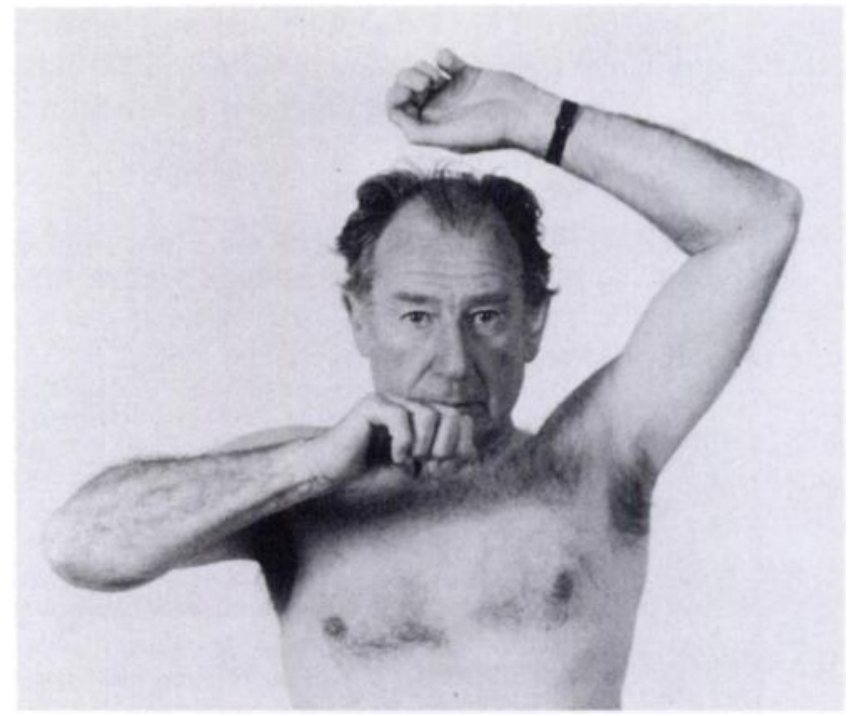

Fig. 1

Case 1. The patient is attempting to flex and laterally rotate his right shoulder.

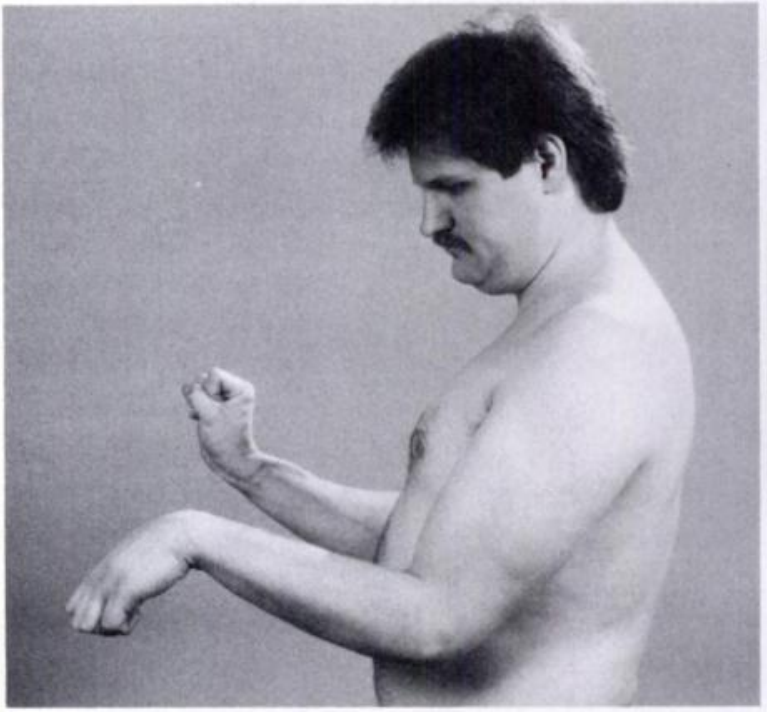

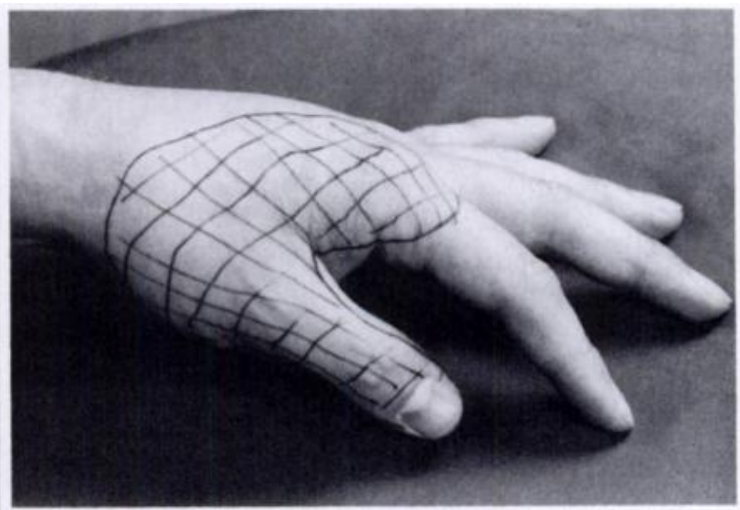

Fig. 3

Fig. 2

Case 2. Figure 2-Profound weakness of extension in the left wrist. Figure 3 - Area of sensory loss (cross-hatched).

\section{DISCUSSION}

Diagnostically, neuralgic amyotrophy must be differentiated from several other causes of shoulder pain including: poliomyelitis, cervical spine disease (whether acute cervical disc disease or cervical spondylosis), primary shoulder disorders (such as impingement syndromes, rotator cuff tears, or calcifying tendinitis), peripheral neuropathies (both compressive and noncompressive) especially entrapment neuropathy of the suprascapular nerve, brachial plexus trauma, and thoracic outlet syndrome.

Biopsy and electrophysiological studies have shown diffuse axonal involvement, rather than focal lesions (Tsairis et al. 1972; Foo and Swann 1983). If the involved nerve is the spinal accessory, the suprascapular, or the long thoracic nerve (all pure motor nerves), and additional sensory changes are observed, then an entrapment neuropathy can be ruled out.

The results of our study paralleled those of others in most respects. We found that a preponderance of cases occurred in men aged 30 to 60 , with significantly more right-sided than left-sided lesions. Patients presented with sudden onset of severe pain (often at night), followed by development of weakness and atrophy as the 
pain subsided. Cases of bilateral involvement were observed. EMG studies often proved helpful in establishing the diagnosis.

Treatment is generally supportive, consisting of analgesics and physiotherapy; braces have not been well tolerated by the patients. Since it is not an entrapment neuropathy, exploration or release of involved nerves is not indicated. Reconstructive surgery may be useful in cases with incomplete recovery after appropriate conservative treatment.

Our study did reveal some features which differ from those previously documented.

1. Pain in the upper extremity is frequently generalised, not confined to the shoulder girdle (as reported by Devathasan and Tong 1980).

2. Involvement of the spinal accessory nerve is relatively common (there were five cases in this study), and may occur as an isolated lesion.

3. There was a $14 \%$ incidence of recurrence in our series, whereas others have reported that recurrence is rare (Tsairis et al. 1972).

4. Clinically demonstrable glenohumeral subluxation, which resolved spontaneously, was found in four of our patients. This is only reported in one other article (Bacevich 1976).

5. Significantly, $64 \%$ of the patients had been seen in orthopaedic consultation during the acute phase of their illness.

Conclusion. Neuralgic amyotrophy is a clinical entity with sudden onset of severe pain and associated sensory abnormalities, reflex changes, and occasional glenohumeral instability. EMG studies are helpful in confirming the diagnosis and treatment is supportive.

\section{REFERENCES}

Bacevich BB. Paralytic brachial neuritis: case report. J Bone Joint Surg [Am] 1976;58-A :262-3.

Bradley WG, Madrid R, Thrush DC et al. Recurrent brachial plexus neuropathy. Brain 1975;98:381-98.

Devathasan G, Tong HI. Neuralgic amyotrophy: criteria for diagnosis and a clinical with electromyographic study of 21 cases. Aust $N Z J$ Med 1980;10:188-91.

Dunn HG, Daube JR, Gomez MR. Heredofamilial brachial plexus neuropathy (hereditary neuralgic amyotrophy with bracial predilection) in childhood. Dev Med Child Neurol 1978;20:28-46.

Flaggman PD, Kelly JJ Jr. Brachial plexus neuropathy: an electrophysiologic evaluation. Arch Neurol 1980;37:160-4.

Foo CL, Swann M. Isolated paralysis of the serratus anterior : a report of 20 cases. J Bone Joint Surg [Br] 1983;65-B:552-6.

James JL, Miles DW. Neuralgic amyotrophy: a clinical and electromyographic study. Br Med J 1966;ii:1042-3.

Lancet. Editorial. Neuralgic amyotrophy: still a clinical syndrome. Lancet 1980;ii:729-30.

O'Brien MD, Payan J. Neuralgic amyotrophy. Lancet 1980;ii :975.

Parsonage MJ, Turner JWA. Neuralgic amyotrophy: the shouldergirdle syndrome. Lancet 1948; : :973-8.

Tsairis P, Dyck PJ, Mulder DW. Natural history of brachial plexus neuropathy: report on 99 patients. Arch Neurol 1972;27:109-17.

Turner JWA, Parsonage MJ. Neuralgic amyotrophy (paralytic brachial neuritis) with special reference to prognosis. Lancet 1957;ii :209-12.

Widerholt WC. Hereditary brachial neuropathy: report of two families. Arch Neurol 1974;30:252-4.

Wyburn-Mason R. Brachial neuritis occurring in epidemic form. Lancet 1941 ;ii :662-3. 